\title{
Actividades para personas mayores y promoción del
}

\section{envejecimiento activo}

Activities for elder people and promotion of active aging

Msc. Nancy Patricia Amador Herrera. ${ }^{1}$ Msc. Julia Barrios Alpízar ${ }^{2}$ Msc. Olivia Margarita Rubio León. ${ }^{3}$ \& Msc. Víctor Manuel González Róbinson. ${ }^{4}$

Recibido: 04-10-2019 / Revisado: 06-11-2019 / Aceptado: 08-12-2019 / Publicado: 03-01-2020

\section{https://doi.org/10.33262/anatomiadigital.v3i1.1109}

\section{Abstract.}

This work belongs to a project with the title Health and Life, integrated for the professors of Physical Education from the Faculty of Medical Sciences Dr. Eusebio Hernández Pérez and 10 students of the medical sciences from the same place. It contributes to the prevention of demential diseases like Alzheimer, in elder people incorporated to the grandparents' house Armenio Ferra Pieselli in the community, from Colón municipality. It counts with activities that help them to establish equilibrium adapted

\section{Resumen.}

Este trabajo pertenece a un proyecto titulado Salud y Vida, integrado por los profesores de Educación Física de la filial Dr. Eusebio Hernández Pérez y 10 estudiantes de las ciencias médicas de la misma, contribuye a la prevención de enfermedades demenciales como el Alzheimer en personas mayores incorporadas a la casa de abuelos Armenio Ferra Pieselli de la comunidad, municipio Colón.

Cuenta con actividades que ayudan a establecer un equilibrio apropiado durante su

1 Filial Medica Dr. "Eusebio Hernández Pérez”. Colon. Matanza, nancypatriciaamador@gmail.com https://orcid.org/0000-0002-7353-0200

${ }^{2}$ Filial Medica Dr. “Eusebio Hernández Pérez”. Colon. Matanza, flor@fcmc.sld.cu iD https://orcid.org/00000002-7353-0201

3 Filial Medica Dr. “Eusebio Hernández Pérez”. Colon. Matanza, orleon@fcmc.sld.cu https://orcid.org/0000-0002-7353-0202

${ }^{4}$ Filial Medica Dr. “Eusebio Hernández Pérez”. Colon. Matanza, vgr@fcmc.sld.cu iD https://orcid.org/00000002-7353-0203 
during their execution. Among them, they represent communicative dynamicand traditional board games, observing movies, videos, listening to music, listening stories and talks. All oriented to the prevention ofdiseases like the Alzheimer in adults. The lofty incidence of the same one in world is enunciated. It is proven that these activities contribute to enlarge the cognoscitive or cerebral reserve that decreases formation of the senile plates that not only contribute to decrease the risks of suffering the disease, but it is an important point against the stress and the depression. Finally, it stands out that there are means valid for all ages through the practice of the different activities with elder people as an aspiration that the long-lived ones know their strengths and weaknesses. Today is a challenge to motivate the grandparents that these are activities used as means of morerecreation, an anti-elect stress, antidepressant and preventive to potentiate an active aging.

Keywords: Activities, aging, health, prevention ejecución, dentro de ellas figuran dinámicas participativas, juegos de mesa, y tradicionales, observar películas, videos, escuchar música, narración de historias, charlas, encaminados a la prevención de enfermedades demenciales como el Alzheimer en las personas mayores, se enuncia la elevada incidencia de la misma en mundo. Se demuestra que estas actividades contribuyen a aumentar la reserva cognoscitiva o cerebral, que disminuye formación de las placas seniles, que no solo contribuyen a disminuir los riesgos de padecer la enfermedad si no que es un medio contra el estrés y la depresión, por último, se destaca que son un medio no farmacéutico vale para todas las edades a través de la práctica de las diferentes actividades con personas mayores se aspira a que los longevos conozcan sus fortalezas y debilidades. Hoy es un reto incentivar a los abuelos a que elijan estas actividades como un medio más de recreación, un antiestrés, antidepresivo y preventivo para potenciar un envejecimiento activo.

Palabras claves: Actividades, envejecimiento, salud, prevención.

\section{Introducción.}

Según la organización mundial de la salud el envejecimiento activo es el proceso de optimización de las oportunidades de salud participación y seguridad, cuyo fin es mejorar la calidad de vida de las personas a medida que envejecen favoreciendo la participación en la comunidad de acuerdo con las necesidades, deseos y capacidades personales.

El Colectivo de autores Manual del animador socio-cultural (2015) expresa que las dinámicas participativas son instrumentos que se utilizan en determinados informativos, consultivos, de toma de decisiones se aplican para adquirir conocimientos partiendo siempre de la práctica de lo que se sabe, de las experiencias vividas y de los sentimientos que muchas originan, así 
como de los problemas y dificultades del entorno facilitan la amenidad, el carácter, el diálogo, alivia tensiones.

Centro San Bartolomé de las casas. [Internet]. (2014) describe que las películas y los vídeos son un excelente vehículo para el aprendizaje impulsa la creatividad y mejora la capacidad para traducir las ideas en palabras, imágenes y sonidos, trasciende lo intelectual y ayuda a reflexionar y expresar posteriormente sus pensamientos y sus ideas de una forma creativa. Son un medio que puede ayudar a que los participantes experimenten otro mundo u otras vidas.

Según Rojas Riveron R. (2007) asegura que la narración de historias es un arte antiguo que consiste en transmitir eventos con palabras y sonidos, a menudo por medio de la improvisación. Por medio de la narración de historias, se pueden desarrollar capacidades para escuchar y para ponerse en los zapatos de los demás. También pueden desarrollar su creatividad y la capacidad de reflexionar sobre su propio comportamiento al penetrar en el mundo que describe la historia.

García Molina M. (2014) nos dice que la música estimula la concentración, la creatividad ayuda a relajarse y reduce los niveles de tensión que inhiben su aprendizaje. Escuchar determinados tipos de música puede desencadenar la liberación de endorfinas y producir un estado tranquilo.

Saramago J. (2016) se refiere a que la poesía, es algo que se hace o se crea, es una forma de arte en la cual se utiliza el idioma por sus cualidades estéticas y evocativas. Los poemas contribuyen a ampliar el significado literal de las palabras y evocan respuestas emocionales a la fantasía o la realidad. Leer poesía aporta cualidades creativas, artísticas y emocionales de una manera que a veces los libros de cuentos no logran trasmitir.

Xiomara N. (2015) argumenta que en el rescate de la memoria lúdica en la persona mayor expresa Los juegos mejoran la capacidad de los participantes para trabajar con los demás, para adquirir confianza en sí mismos, para descubrir nuevas ideas y para cuestionar sus prejuicios, son apropiados en el aprendizaje a través de la educación ética. Sin embargo, es necesario crear un entorno propicio para que se produzca una competencia justa y respetuosa y evitar juegos en donde se presentan "perdedores" y "ganadores.

Pérez Martínez, V. (2005) plantea que la demencia en la enfermedad del Alzheimer se explica que enfermedad de Alzheimer fue descrita por el neuropatólogo Alemán Alois Alzheimer en 1906, quien siguió el curso clínico de una mujer. El Alzheimer es una enfermedad degenerativa cerebral primaria, un probable síndrome multifactorial en su origen, heterogéneo en su clínica, en su neuropatología y en la predisposición genética. Es un trastorno exclusivo del sistema nervioso central en el que se produce una lenta destrucción y 
atrofia de la corteza cerebral, y se deposita un material fibrilar de características amiloides en forma de haces neurofibrilares, placas neríticas e infiltrados vasculares.

Como consecuencia de ello, aparece una alteración progresiva de las funciones intelectuales, que puede comenzar por trastornos aparentemente leves de memoria, pero que desemboca en una demencia grave de tipo cortical. El curso y manifestaciones clínicas pueden ser muy variables. Su diagnóstico definitivo se basa en la comprobación anatomopatológica de la presencia de haces neurofibrilares y placas neuríticas en una densidad determinada, pero se puede establecer un diagnóstico probable basado en la clínica y en la evaluación neuropsicológica En el mundo hay 46,8 millones de personas que padecen demencia y entre el 60\% y $70 \%$ de esos casos corresponden a Alzheimer. Se estima que para el año 2050 el número ascienda a 131.5 millones de personas con este trastorno.

Muñoz Chacón Y. (2010) plantea que, en Cuba, de acuerdo con investigaciones realizadas, se estiman alrededor de 130 mil las personas que padecen Alzheimer o una demencia relacionada, y este número se incrementará en 2,3 veces para el 2040, es decir a 300 mil personas con demencia, lo que representará para esa fecha el 2,7\% de nuestra población, si no se logra alcanzar una cura efectiva para la enfermedad.

La demencia en la enfermedad se inicia por lo general de manera insidiosa, evoluciona lenta y progresivamente durante años. Aunque puede aparecer en la edad madura de la vida, o incluso antes, su incidencia es mayor hacia el final de la vida, por lo que su presentación y evolución clínica son muy variables. Posee rasgos neuropatológicos y neuroquímicos característicos.

Según Vidal Rivas L. (2016) la charla no es más que charlar, hablar acerca de un tema que se en un ambiente familiar, distendido y ameno sin solemnidad o formalidad habitual.

Las diferentes investigaciones realizadas, unido a las vivencias y realidades cotidianas nos revelan que el gran volumen de tiempo libre del que disponen los abuelos no resulta ocupado y aprovechado de la mejor forma en función de la satisfacción de las necesidades recreativas y la salud existiendo insuficiencias en la realización de programaciones recreativas, las cuales carecen en su mayoría de variedad, trayendo consigo la insatisfacción de la población y por tanto, una creciente demanda de este tipo de actividades, por lo que aún son insuficientes las actividades físicas-recreativas para la ocupación del tiempo libre del adulto mayor del consejo popular Este, lo que constituye la situación problémica que orienta el presente trabajo.

Lo que estimuló a las autoras a planificar y realizar actividades físicas-recreativas que contemplen los gustos y preferencias y la utilización de algunas acciones cotidianas que realizan las personas mayores incorporadas a la casa de abuelos Armenio Ferra Pieselli de la 
comunidad, municipio Colón este. Teniendo como objetivo: Potenciar el bienestar físico, social y mental a lo largo del ciclo vital para una mejor calidad de vida.

\section{Desarrollo}

\section{Material y Métodos.}

Se realizó un estudio observacional, descriptivo propositivo y de intervención, se revisaron y valoraron los contenidos de las orientaciones metodológicas del programa de recreación física para el adulto mayor y las actividades fundamentales para su vinculación con algunas acciones cotidianas que realizan las personas de la tercera edad vinculadas con el mantenimiento de las capacidades coordinativas y condicionales que se trabajan con el objetivo de obtener la información requerida para conformar el material de las actividades físicas-recreativas propuestas. Además de la consulta con especialistas de la cultura física, la recreación física y salud pública que aportaron diferentes ideas y variantes para la confección de las mismas.

Fueron analizados y comparados diferentes materiales que contenían juegos y otras actividades teniendo en cuenta los gustos y preferencias de estas personas para utilizarlas en su recreación que permitieron conformar el material de actividades físicas-recreativas para contribuir a mejorar la ocupación del tiempo libre del adulto mayor.

\section{Población y Muestra.}

La casa de abuelos Armenio Ferra Pieselli de la comunidad, municipio Colón Este tiene una cantidad de 40 abuelos de estos 39actos para realizar ejercicio físico.

Se aplicó encuesta con el objetivo de recoger información acerca del uso de las actividades físicas-recreativas en su tiempo libre y su satisfacción en las mismas para la confección del material arrojando la siguiente información.

Los abuelos encuestados el 97,5\% respondió que si realizan actividades físicas - recreativas en su tiempo libre, la mayoría coincidió en que las actividades que realizan en tu tiempo libre son juego de dominó, entre otros, las actividades cotidianas que realizan con más frecuencia durante el día la mayoría también coincidió con las que se reflejan a continuación: Comprar la leche y el pan, hacer los mandados en la bodega, ir al mercado (placita), llevar y recoger los nietos al círculo, entre otras, manifestaron que no realizan actividades físicas recreativas vinculadas con acciones cotidianas y que les gustaría en su tiempo libre realizar actividades físicas-recreativas vinculadas con las acciones cotidianas que realiza frecuentemente.

Se aplica entrevista a 5 profesores de cultura física y de recreación de los 5 profesores entrevistados, nos arroja un resultado que el $100 \%$ de los entrevistados no trasmiten a sus 
abuelos/as sistemáticamente actividad físicas-recreativas vinculadas con las acciones cotidianas que ellos realizan, y que cuentan con poco material que puedan vincular con acciones cotidianas que realiza el adulto mayor que le permita orientarlo(a), esto nos da a entender que es de suma importancia que los profesores de cultura física cuenten con herramientas de trabajo que nos ayuden a contribuir a que las clases sean más dinámicas y motivadas en nuestros abuelos/as

\section{Resultados.}

La incorporación de los abuelos/as debe ser paulatina y voluntaria, no se debe imponer la realización de las actividades. Para ello, el profesor utilizará una motivación sugerente y cercana a los mismos. Para la planificación de las diferentes actividades se tuvo en cuenta los tres objetivos ligados al envejecimiento activo.

1. Bienestar físico esto puede favorecer la nutrición y el ejercicio físico.

2. Bienestar psíquico-cognitivo emocional este rubro incluye una serie de actividades para favorecer la plasticidad cerebral y las funciones cognitivas.

3. Bienestar socio-emocional sus funciones favorecer las relaciones cordiales, aumentar las relaciones interpersonales ayuda a contrarrestar sensaciones de soledad.

Las actividades son una gimnasia mental que contribuyen a aumentar las facultades intelectuales, son puramente lógicas donde el razonamiento y la comprensión son factores indispensables para el buen desarrollo de la memoria, el cálculo y la concentración. Resultan un instrumento incomparable por lo que se le llama aprender a aprender favorece el ejercicio y desarrollo de varias habilidades mentales además de la formación del pensamiento científico, ya que quienes participan se someten a una constante puesta a prueba de hipótesis que deben verificar o descartar.

\section{Metodología}

Dinámica: Deje aquí su carga pesada ... y recoja alegría.

Objetivo: animación.

Desarrollo: Antes de entrar al local, cada participante toma dos tarjetitas en blanco y un pequeño obsequio. Luego en la tarjetita en blanco escribe cuál es su carga pesada. Arruga el papel, sopla sobre él y lo coloca en la javita. A continuación, escribe en la otra tarje titaun mensaje que genere alegría para la persona a quien se lo va a entregar. Al final expresan como se han sentido se han seleccionado otras dinámicas el semáforo, las sillas peligrosas, el navío mercante, carrera de limones, lluvia de ideas, como el papel, bálsamo para los deprimidos, completar frases, refranes incompletos, los corazones, el pañuelo risueño, etc. 
Película: El Benny

Video: Toni Meléndez.

Objetivo: Elevar autoestima.

Desarrollo: Antes hacer un breve resumen del argumento de la película o video y del período en que se sitúa. Preguntar por qué cree que ver esta película es importante. Otras sugerencias de películas y videos El circo de las mariposas, Kirifu y la bruja, Ponientes, Diamante de sangre, El bola, Gandhi, Cadena de favores, Diario de motocicletas, El viaje, María llena eres de gracias, Ciudad de Dios, La vendedora de rosas, Diarios de la calle Oliver Twist etc. La paz Los valores pueden salvar la humanidad, ALBA-ALBA-ALBA Life VestInsideKindness Boomerang One Day, Mira la vida con ojos nuevos, Nuevo día, Sigue tocando, Vocación de The Potter, Cuando me ame de verdad, Hoy morí de estrés, Volver a ser feliz, Toni Meléndez, Los marcianos Celebra la vida, Corto cuerda, Relájate escucha y admira, Para disfrutar, Más tiempo, Reflexión el corcho Como el lápiz, No te rindas, etc.

\section{Poema: La solidaridad.}

Objetivo: Fomentar un momento de reflexión y silencio para que sean capaces de expresar los sentimientos del autor, así como que valores se ponen de manifiesto.

Desarrollo: Antes de dirigirse al lugar acordado hacer un breve resumen del argumento del poema y del período en que se sitúa. Su autor país, etc. Se han seleccionado una lista de poemas que pueden ayudar al tema en cuestión. Que haya paz, Mi corazón es capaz, Dos consejos El agradecimiento en lo pequeño, Lemas, El coraje y la libertad, Sal para el alma, De todos modos, Anoche cuando dormía, Piececito, Estados de ánimos, Hagamos un trato, Enseñanza sobre la bondad.

Narración de historia: El problema del Sultán.

Objetivo: Desarrollarla capacidad de escuchar y de conectarse unos con otros entrando juntos en el mundo de los cuentos de abrirse al mundo de la imaginación.

Desarrollo: Crear un entorno adecuado para contar cuentos. Puede ser alrededor de una hoguera, en un parque donde estén en contacto con la naturaleza o en un lugar tranquilo. Para comenzar la narración puede emplear velas, incienso, instrumentos musicales como una guitarra o unos bongos, o poner música tranquil

Sugerencias, Los tres ciegos, La zorra y la cigüeña, Las tres plumas, La amistad, Estrella de mar, ¿La dama o el tigre? El anillo, Las cebollas, La palmera del desierto, La huelga de las palabras. 
Juego: Cada oveja con su pareja.

Objetivo: Fomentar la capacidad de trabajar en equipo y de construir puentes de confianza entre los participantes en aras de un objetivo común.

Desarrollo: Este juego consiste en agrupar todos elementos de una misma categoría que se presentan mezclados encima de una mesa pueden ser botones por colores por tamaño fichas legumbres entre otras sugerencias La tela de araña, Todos a él o a ella, El baile de las sillas, Tú silueta es la mía, Alcanzar las estrellas, Reconocimiento gozoso, Cómo sería el mundo si..., El árbol de la vida, Semanas temáticas, Creación de proyectos, Reflexión sobre el respeto, Situaciones injustas, etc.

\section{Música: Cha cha cha}

Objetivo: Sensibilizar para que se expresen y se escuchen los unos a los otros, dialoguen y compartan ideas entre ellos y se relacionen de una manera respetuosa, autentica, libre.

Desarrollo: Antes hacer un breve resumen del período en que se sitúa la música a escuchar. Preguntar por qué cree que escuchar música es importante Sugerencia. A Daniel, un chico de la guerra Alberto Cortes, Color esperanza Diego Torres, Todo cambia, Gracias a la vida, Mercedes Sosa, Fíjate bien, Bandera de manos Juanes, Derecho humano Silvio Rodríguez, Cantare cantara varios países Yo viviré Celia Cruz, La muralla, Ana Belén y Víctor M. El Niágara en bicicleta Juan Luis Guerra, Yo soy rebelde Rosario Flores, Mas allá, Puedes llegar Gloria Stefan, Fe Mana etc. Estudios han demostrado que las personas mayores que tienen pasatiempos y que utilizan su cerebro de manera activa corren dos veces menos el peligro de padecer Alzheimer.

\section{Charla: La nutrición.}

Objetivo: Promover una alimentación saludable.

Desarrollo: Se introduce el tema y se da a conocer como debe ser la alimentación de un adulto horarios, llevarse a la boca pequeñas porciones se debe sustituir por legumbres, por pescado huevos, consumir huevo dos veces por semanas, aumentar el consumo rico en fibras frutas, verduras crudas, pan y cereales integrales, lácteos dos veces al día, beber agua 6 a 8 vasos diarios, se concluye intercambiando con los abuelos. Sugerencias para otros encuentros el baño del adulto mayor, higiene bucal, medicina verde, prevención de caídas, salud mental entre otras.

\section{Conclusiones.}

- El Alzheimer. Es un trastorno exclusivo del sistema nervioso central en el que se produce una lenta destrucción y atrofia de la corteza cerebral, y se deposita un 
material fibrilar de características amiloides en forma de haces neurofibrilares, placas neríticas e infiltrados vasculares la causa principal de la enfermedad es la acumulación de proteínas amiloides, se manifiesta principalmente en las personas mayores. Hasta el momento no existe una cura.

- Las diferentes actividades contribuyen a la formación de reservas cognitivas, la mejor opción no farmacéutica para prevenir el Alzheimer, no solo previene la enfermedad si no que contribuye de forma activa y lozana a un mejor desenvolvimiento social del individuo elevan cociente intelectual, ejercita ambos hemisferios cerebrales, mejora la creatividad, potencia la memoria.

- Las actividades son una gimnasia mental que contribuyen a aumentar las facultades intelectuales, son puramente lógicas donde el razonamiento y la comprensión son factores indispensables para el buen desarrollo de la memoria, el cálculo y la concentración

\section{Referencias bibliográficas.}

Colectivo de autores. (2015). Manual del animador socio-cultural Lo que tengo te lo doy vol.2 La Habana Cuba.abacoenred.com > 2016/04 > Manual-para-la-ASC-2010Chiapas.pdf.pdf

Centro San Bartolomé de las casas. (2014). [Internet] Que la pelota no toque el suelo. Fundación Arigatou [citado 5 mayo 2015]; 6(2):115. Disponible en: http://www.ethicseducationforchildren.org

Rojas, R. R. (2007)El Ungüento de la Magdalena ediciones la memoria Centro Cultural Pablo de la Torriente Brau premio.

www.lajiribilla.cu > articulo > mas-ungueento-de-la-magdalena

García, M. M. (2014).La importancia de la música en el adulto mayor

https://rodin.uca.es > xmlui > bitstream > handle

Saramago, J. (2016).Un poema sobre la vejez https://www.elsiglodetorreon.com.mx > blogs $>$ HYPERLINK

"https://www.google.com/url?sa=t\&rct=j\&q=\&esrc=s\&source=web\&cd=1\&cad=rj a\&uact=8\&ved=2ahUKEwiVuK6MrqjkAhXKtlkKHWJaCAwQFjAAegQIAxAB\& url=https $\% 3 \mathrm{~A} \% 2 \mathrm{~F} \% 2 \mathrm{Fwww}$.elsiglodetorreon.com.mx $\% 2 \mathrm{Fblogs} \% 2 \mathrm{Fmottivare} \% 2 \mathrm{~F}$ 2201-un-poema-sobre-la-vejezjose\&usg=AOvVaw36dkQ14T2kchyv7BiyXYCm"mottivareHYPERLINK "https://www.google.com/url?sa=t\&rct=j\&q=\&esrc=s\&source=web\&cd=1\&cad=rj 
a\&uact=8\&ved=2ahUKEwiVuK6MrqjkAhXKtlkKHWJaCAwQFjAAegQIAxAB\& url=https $\% 3 \mathrm{~A} \% 2 \mathrm{~F} \% 2 \mathrm{Fwww}$.elsiglodetorreon.com.mx\%2Fblogs\%2Fmottivare $\% 2 \mathrm{~F}$ 2201-un-poema-sobre-la-vejez-jose\&usg=AOvVaw36dkQ14T2kchyv7BiyXYCm" , 2201-un-poem..

Xiomara, N. (2015).En el rescate de la memoria lúdica en la persona mayorhttps://repository.libertadores.edu.co

Pérez, V.T.(2005). Demencia en la enfermedad de Alzheimer: un enfoque integral. Rev. Cubana MGI v.21 n 3-4.Ciudad de la Habana mayo-agosto

Muñoz, Ch. Y. (2003). Demencia, el reto del presente siglo (artículo completo disponible en español). Acta méd. Costarric. [Online]. vol.45, no.2 [Consultado el 2 de enero de 2010], p.42-42. ISSN 0001-6002

www.scielo.sa.cr > scielo > pid $=\mathrm{S} 0001-60022003000200002$

Vidal, R. L.(2016).Deficion de una charla (artículo completo disponible en español).https://www.youtube.com/watch

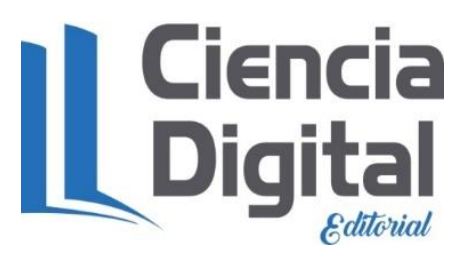


PARA CITAR EL ARTÍCULO INDEXADO.

Amador Herrera, N. P., Barrios Alpízar, J., Rubio León, O. M., \& González Róbinson, V. M. (2020). Actividades para personas mayores y promoción del envejecimiento activo. Anatomía Digital, 3(1), 6-16. https://doi.org/10.33262/anatomiadigital.v3i1.1109

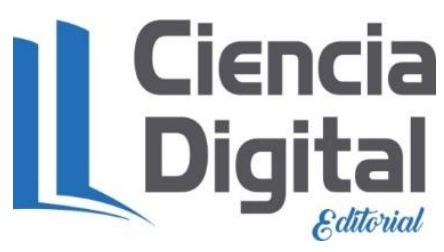

El artículo que se publica es de exclusiva responsabilidad de los autores y no necesariamente reflejan el pensamiento de la Revista Anatomía Digital.

El artículo queda en propiedad de la revista y, por tanto, su publicación parcial y/o total en otro medio tiene que ser autorizado por el director de la Revista Anatomía Digital.
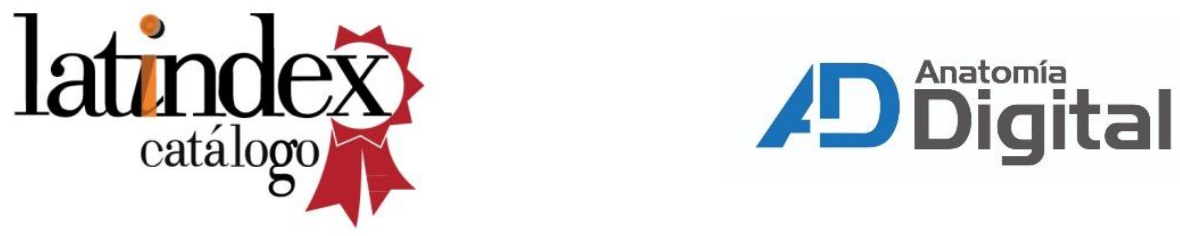